\title{
Epidemiological study of klebsiella infection in the special care baby unit of a London hospital
}

\author{
EVE RISER, PAUL NOONE, AND FRANCES M HOWARD \\ From the Departments of Medical Microbiology and Paediatrics, Royal Free Hospital, Pond Street, \\ Hampstead, London NW3 $2 Q G, U K$
}

SUMmaRY Of the babies admitted to the Special Care Baby Unit of the Royal Free Hospital over 20 months, $10 \cdot 2 \%$ were infected or colonised by klebsiella. The fluorescent antibody technique was used to identify epidemics caused by three strains: capsular type $8 \mathrm{~K}$. aerogenes, type $68 \mathrm{~K}$. oxytoca, or type $13 \mathrm{~K}$. aerogenes, each of which was predominant at a different time, exhibited a difference in virulence, and showed a predilection for different sites of infection. Intestinal colonisation was frequently followed by the presence of sepsis in other sites by the same capsular type. Antibiotic administration led to a higher incidence of klebsiella infection, while the widespread use of compounds containing hexachlorophane could have contributed to skin colonisation and infection by klebsiella. An environmental survey indicated that $1 \%$ Hycolin failed to disinfect the incubators, that the babies were the reservoirs of the organisms, and that transmission was due to inadequate handwashing of nurses and mothers. The mothers were found to have been uninformed of hygienic techniques. They were observed in various practices which could have contributed to the spread of the organism, including contaminating communal areas and handling babies other than their own. It has been recommended that the mothers of premature infants be instructed in the hygienic measures required in dealing with this susceptible population and that the nursing and medical staff be more strict in their own observance of these procedures.

Epidemics caused by klebsiella in special care baby units have been previously reported by Hable $e t$ al. (1972), Cichon et al. (1977), and Pierog et al. (1977).

The New Royal Free Hospital in London was opened to admit patients in October 1974. Strains of klebsiella isolated from routine specimens were collected and typed by capsular fluorescence. This offered a unique opportunity to monitor the occurrence and dissemination of different types of klebsiella as they first appeared in the new hospital environment.

During the following year, random types were isolated from the hospital in general with little sign of duplication of types within individual wards. The Special Care Baby Unit (SCBU) yielded scattered isolations of types 9,19 , and 33 from umbilical swabs, incubators, and extractor fans but without any indication of serious cross-infection.

Received for publication 15 October 1979
In January 1976, capsular type 8 was isolated from a nose swab of a baby in the SCBU. This particular type continued to recur and was recovered from various sites of 46 premature infants during the next 16 months.

As the entire hospital had been under surveillance for repeating types this recurrence was noted in its early stage, and after three months the numbers suggested a possible outbreak in this ward. Therefore, a thorough study of the environment of the SCBU was planned to establish whether an epidemic existed and, if so, to identify the source of infection or to explain by what other means this particular strain was being transmitted from one infant to another.

\section{Material and methods}

\section{FLUORESCENCE TYPING}

The fluorescent antibody technique, as developed by Riser et al. (1976) for typing klebsiella, was used to determine the capsular types of all the isolates in this study. 


\section{ROUTINE ISOLATIONS}

Specimens were taken routinely from the eyes, ears, nose, throat, umbilicus, and rectum of each baby on admission to the SCBU. Thereaf er specimens were taken if there was an indication of infection in any particular site. Organisms isolated and identified as Klebsiella species (Cowan, 1974) were maintained on nutrient agar slopes (Southern Group Laboratories) until typed.

\section{ENVIRONMENTAL SURVEY}

In May 1976, a survey was made of the non-living environment, the personnel, and all the babies in the unit at that time. This included investigation of the hot $\left(25.5^{\circ} \mathrm{C}\right)$ and cold $\left(24^{\circ} \mathrm{C}\right)$ nurseries, the milk room, storage room, and adjoining treatment rooms.

Swabs were taken of surfaces, equipment, disinfectants, feeds, and materials used on the babies. Throat and rectal swabs were obtained from the doctors, nursing staff, and parents of the babies, as well as from all the infants in the unit whether exhibiting an active infection or not. The swabs were inoculated either directly on to MacConkey agar plates (Oxoid) and blood agar plates (nutrient agar, Southern Group Laboratories; 5\% horse blood, Tissue Cultures Ltd) and incubated at $37^{\circ} \mathrm{C}$ or first into Robertson's cooked meat medium, incubated at $37^{\circ} \mathrm{C}$, then plated on to MacConkey's and blood agar plates and incubated at $37^{\circ} \mathrm{C}$.

\section{EXAMINATION OF HANDS}

Because of its high efficacy and simplicity, the fingertip procedure was the technique used in determining hand carriage of klebsiella in this study.

Finger impression cultures were taken from nurses and mothers after they had changed a baby, before and after washing their hands with Betadine or Hibiscrub. The fingertips of both hands were touched on to MacConkey agar plates, which were then incubated at $37^{\circ} \mathrm{C}$.

\section{EXAMINATION OF DISINFECTANTS}

The in-use test of Kelsey and Maurer (1966) was used for detection of contaminated disinfectants. Tween 80 was used to neutralise Hycolin, Betadine, chlorhexidine, and Hibiscrub.

EXAMINATION OF CLEANED INCUBATORS Incubators and cots in both the hot and cold nurseries were swabbed routinely after being cleaned with $1 \%$ Hycolin, and the swabs were sent to the laboratory for culture. A nine-month period was selected to evaluate the efficiency of the disinfectant. During this time there was a high isolation rate of type 8 klebsiella from the babies.
ASSESSMENT OF CLINICAL SIGNIFICANCE OF KLEBSIELLA ISOLATES

All the specimens from the babies were assessed by an experienced paediatrician (FMH) in the unit as to whether the presence of klebsiella was of clinical significance. The isolates were divided into three groups based on the correlation of signs and symptoms and relevant tests including laboratory reports.

\section{Clinically significant}

The baby appeared clinically ill, usually with skin, respiratory, or alimentary symptoms and was treated with antibiotics for klebsiella.

\section{Possibly significant}

The baby appeared well and behaved normally, or sepsis was obviously due to another organism.

\section{Not assessed}

The data available were either insufficient to attribute sepsis to klebsiella or to determine the degree of relevance of the presence of the organism.

\section{EFFECT OF ANTIBIOTICS}

It was noted whether babies had received antibiotics before they became colonised or infected with a klebsiella.

\section{Results}

\section{ENVIRONMENTAL SURVEY}

Over 150 samples were taken of the environment, personnel, and parents of the babies in the SCBU. Although many other genera of microorganisms were recovered from this survey, type 8 klebsiella was found only in an incubator, in a cot, and on the ledge of an inset light unit open to the ceiling area above the hot nursery; but it could not be reisolated from the latter on successive attempts or by use of settle plates left in the light unit or in the space above the ceiling. Table 1 lists the environmental sources examined, the number of samples from each general source, the amount of growth of klebsiella on MacConkey or blood agar plates, and the capsular type of the klebsiella isolated.

\section{EXAMINATION OF HANDS}

A total of 23 hand samplings were taken. Of these, eight nurses and two mothers $(43.5 \%)$ had klebsiella or an enterobacter on the hands after changing a baby. The klebsiella was shown to be the same capsular type as that infecting the corresponding baby. Three nurses $(30 \%)$ still had the same organisms on their hands after washing with Betadine or Hibiscrub (Table 2).

The hands of three nurses were tested before they 
Table 1 Number of environmental samples from different sources, amount of growth of klebsiella on MacConkey or blood agar plates, and the capsular type of the klebsiella isolated

\begin{tabular}{|c|c|c|c|}
\hline Source & $\begin{array}{l}\text { Number of } \\
\text { samples }\end{array}$ & Growth of klebsiella & $\begin{array}{l}\text { Capsular } \\
\text { type }\end{array}$ \\
\hline Sink & 20 & - & \\
\hline Sluice sink & 1 & - & \\
\hline Vitamins & 6 & - & \\
\hline Betadine & 12 & - & \\
\hline Outlets & 5 & - & \\
\hline Inset light unit & 2 & $\because-\cdots$ to - (repeat) & 8 \\
\hline Milton & 2 & - & \\
\hline Hibiscrub & 7 & - & \\
\hline Suction tubing & 1 & - & \\
\hline Resusitair tubing & 1 & - & \\
\hline Eye drops & 1 & - & \\
\hline Inlets & 6 & - & \\
\hline Settle plate & 5 & - & \\
\hline Chlorhexidine & 9 & - & \\
\hline Glycerin & 3 & - & \\
\hline Mop & 1 & - & \\
\hline Sodium hypochlorite & 1 & - & \\
\hline Phisohex & 2 & - & \\
\hline \multicolumn{4}{|l|}{ Hand cream } \\
\hline (chlorhexidine) & 16 & - & \\
\hline (other) & 1 & - & \\
\hline Soap dish & 1 & - & \\
\hline Salt & 2 & - & \\
\hline \multicolumn{4}{|l|}{ Zinc and castor oil } \\
\hline cream & 3 & - & \\
\hline \multirow[t]{2}{*}{ Cot } & 9 & - & \\
\hline & 1 & 2 col to - (repeat) & 8 \\
\hline \multirow[t]{2}{*}{ Incubator } & 9 & - & \\
\hline & 1 & $1 \mathrm{col}$ & 8 \\
\hline Extractor & 2 & - & \\
\hline Hycolin & 3 & - & \\
\hline Bath & 1 & - & \\
\hline Splint & $i$ & - & \\
\hline Paraftin & 1 & - & \\
\hline Trolley top & 1 & - & \\
\hline Systan cream & 1 & - & \\
\hline Ster-zac powder & 1 & - & \\
\hline Milk & 1 & - & \\
\hline Dextrose & 1 & - & \\
\hline Scales & 1 & - & \\
\hline Table top & 2 & - & \\
\hline Injection swab & 1 & - & \\
\hline
\end{tabular}

changed the babies and no klebsiella was isolated. One of these nurses acquired the type of the baby she then changed, but the organisms were no longer detected after the hands had been washed. A control baby who was not colonised with klebsiella was included. Klebsiella was not present on the hands of the nurse before she changed this baby, after the baby was changed, or after the hands had been washed. The number of colonies on the plates was recorded in three instances and showed moderate growth from the fingertips after colonised or infected babies had been changed. This amount was either sharply reduced or eliminated in these cases when the hands had been washed.
EXAMINATION OF INCUBATORS

Two hundred and four post-cleaning incubator swabs had been taken during the nine months selected to test the efficiency of $1 \%$ Hycolin in disinfecting the incubators. Ninety-six $(47 \cdot 1 \%)$ of the swabs grew various microorganisms; $24(11 \cdot 8 \%)$ yielded Klebsiella species, and $16(7 \cdot 8 \%)$ klebsiella capsular type 8 .

\section{ASSOCIATION OF SEROTYPES WITH SEPSIS}

The most common site of isolation of type 8 was the skin, especially the umbilicus, and comprised $40 \%$ of the type 8 isolates. The respiratory tract accounted for $34 \%$ and the intestine for $13.8 \%$. Of the isolates of this type, $18.5 \%$ were responsible for sepsis, $32.3 \%$ for a possible infection, $32.3 \%$ for colonisation, and $16.9 \%$ could not be assessed (Table 3 ).

Type 68 was isolated from an incubator on 9 July and from a baby on 10 July 1976. A few other random types were recovered during the next few months, but type 8 was still the predominant organism although the number of new cases was declining There was a short gap in the occurrence of new klebsiella isolations when type 68 suddenly became the major organism, being accompanied by another rise in the incidence of type 8 .

Table 3 shows that two $(13.3 \%)$ type 68 were responsible for definite infections, seven $(46.7 \%)$ for possible infections, and six $(40 \%)$ for colonisation. Type 68 was found most frequently in stool specimens $(40 \%$ of this type).

The two-month period during which type 68 became predominant and type 8 reappeared coincided with an outbreak of necrotising enterocolitis that occurred in the unit during January and February 1977.

Types 8 and 68 disappeared after the unit had been closed for one month because of the outbreak of necrotising enterocolitis. Only one more isolation of type 8 was recorded, a month after the SCBU had been reopened. After this there were two cases of type 3 and two of type 18 . But these were soon replaced by type 13 , which became the predominant organism and occurred in 12 different neonates over the next three months. It was still present when the study ended.

The number of isolates of type 13 and their clinical relevance are listed in Table 3. None of the isolates was positively associated with sepsis although nine $(50 \%)$ may possibly have been. Seven $(38.9 \%)$ were probable colonisations while two $(16.1 \%)$ were unassessed.

The progression of the number of new cases and the causative capsular types are indicated in the Figure. It can be seen that one type generally 
Epidemiological study of klebsiella infection in the special care baby unit of a London hospital

Table 2 Klebsiella on nurses' and mothers' hands after handling babies

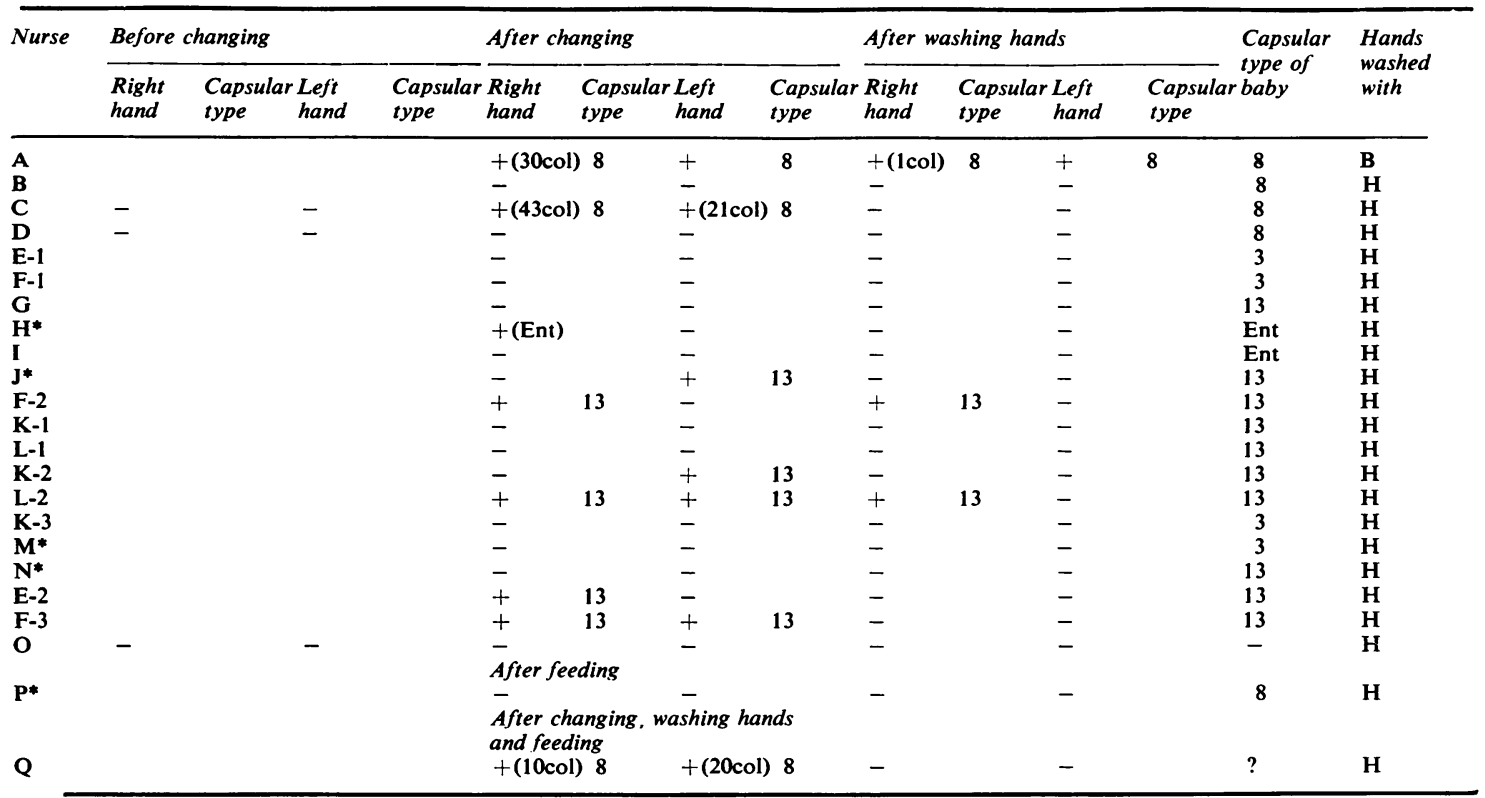

*Mother; + klebsiella isolated col $=$ number of colonies on plate; $\mathbf{B}=$ Betadine; $\mathbf{H}=$ Hibiscrub; Ent $=$ enterobacter.

Table 3 Number of isolates and percentage of each capsular type from different neonatal sources and their clinical relevance

\begin{tabular}{|c|c|c|c|c|c|}
\hline \multirow[t]{2}{*}{ Specimen } & \multirow[t]{2}{*}{ Capsular type } & \multicolumn{4}{|c|}{ Clinical relevance } \\
\hline & & Significant & Possibly significant & Not relevant & Not assessed \\
\hline \multirow[t]{2}{*}{ Respiratory } & $\begin{array}{r}8 \\
68\end{array}$ & $2(3 \cdot 1)$ & $\begin{array}{ll}5 & (7 \cdot 7) \\
1 & (6 \cdot 7)\end{array}$ & $\begin{array}{l}9(13 \cdot 8) \\
4(26 \cdot 7)\end{array}$ & \\
\hline & 13 & & $2(11 \cdot 1)$ & $2(11 \cdot 1)$ & $1 \quad(5 \cdot 6)$ \\
\hline \multirow[t]{2}{*}{ Skin } & 8 & $7(10 \cdot 8)$ & $11(16.9)$ & $5(7 \cdot 7)$ & $3 \quad(4 \cdot 6)$ \\
\hline & 68 & $1(6 \cdot 7)$ & $2(13 \cdot 3)$ & $1(6 \cdot 7)$ & \\
\hline \multirow{2}{*}{ Urinary } & $\begin{array}{r}13 \\
8\end{array}$ & $1 \quad(1.5)$ & $3(16 \cdot 7)$ & $4(22 \cdot 2)$ & $1 \quad(5 \cdot 6)$ \\
\hline & $\begin{array}{l}68 \\
13\end{array}$ & & $\begin{array}{ll}1 & (5 \cdot 6)\end{array}$ & & \\
\hline \multirow{2}{*}{ Rectal } & 8 & & $3 \quad(4 \cdot 6)$ & $5 \quad(7 \cdot 7)$ & $1 \quad(1.5)$ \\
\hline & $\begin{array}{l}68 \\
13\end{array}$ & $1(6 \cdot 7)$ & $\begin{array}{l}4(26.7) \\
3(16.7)\end{array}$ & $\begin{array}{ll}1 & (6.7) \\
1 & (5.6)\end{array}$ & \\
\hline \multirow[t]{2}{*}{ Blood } & $\begin{array}{r}13 \\
8 \\
68\end{array}$ & $1(1.5)$ & $3(1007)$ & & \\
\hline & 13 & & & & \\
\hline Tubing & $\begin{array}{l}8 \\
68 \\
13\end{array}$ & $1 \quad(1 \cdot 5)$ & $1(1 \cdot 5)$ & & $1 \quad(1 \cdot 5)$ \\
\hline Total & $\begin{array}{r}13 \\
8 \\
68 \\
13\end{array}$ & $\begin{array}{r}12(18.5) \\
2(13.3)\end{array}$ & $\begin{aligned} 21 & (32 \cdot 3) \\
7 & (46 \cdot 7) \\
9 & (50)\end{aligned}$ & $\begin{aligned} 21 & (32 \cdot 3) \\
6 & (40) \\
7 & (38 \cdot 9)\end{aligned}$ & $\begin{array}{r}11(16 \cdot 9) \\
2(11 \cdot 1)\end{array}$ \\
\hline
\end{tabular}

Figures in parentheses are percentages. 


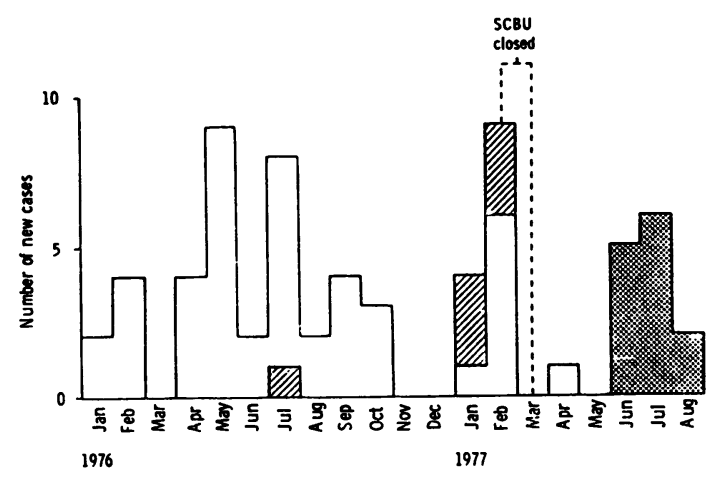

Occurrence of type $8 \square$, type $68 \mathrm{ZT}$, and type 13 in the Special Care Baby Unit.

predominated at a time, eventually being replaced by another type.

EFFECT OF ANTIBIOTICS

After 12 babies had received antibiotics, six $(50 \%)$ developed a definite sepsis and were treated for klebsiella; three had a possible infection but were not treated. Of 60 neonates not receiving antibiotics, only five $(8.3 \%)$ developed a definite sepsis, which was treated for klebsiella, and 21 a possible sepsis, which was not treated.

\section{Discussion}

Seventy-two out of $705(10 \cdot 2 \%)$ of the babies admitted to the Special Care Baby Unit of the Royal Free Hospital during a continuous 20-month period were found to be infected or colonised by a klebsiella. The fluorescent antibody technique showed that the presence of klebsiella was due primarily to sequential epidemics caused by three capsular types.

Type $8 \mathrm{~K}$. aerogenes accounted for $43.3 \%$ of the klebsiellae isolated; $18.5 \%$ of this type was attributed to disease and $10.8 \%$ caused skin infections.

Type $68 \mathrm{~K}$. oxytoca comprised $10 \%$ of the klebsiellae found, $13.3 \%$ of this type causing sepsis of the gut or skin. If the figures for 'possible significance' are also considered, this strain may be potentially more pathogenic for the intestine while it is merely a commensal in the respiratory tract.

Twelve klebsiellae were type $13 \mathrm{~K}$. aerogenes. None of this type was definitely associated with disease but may possibly have caused skin or gastrointestinal infections; but many of the skin isolates were commensals.

Type 8 thus appears to be more virulent than type
68 , which may be more virulent than type 13 . A difference in virulence related to serotype has also been noted by other workers. Pierog et al. (1977) reported an epidemic of neonatal septicaemia of unusually low virulence due to $K$. pneumoniae type 60. Virulence studies in mice showed that $K$. pneumoniae type 33 isolated from blood and tracheal aspirates was considerably more virulent than klebsiellae types 11,19 , and 30 from the same sites in other patients (Hable et al., 1972).

Type 8 was more common in skin isolates while type 68 was isolated most frequently from faeces. Type 13 is probably a commensal on the skin but could possibly have been pathogenic in the intestine. This suggests a preference of different types for a particular site, especially a site in which the organism could possibly be more pathogenic. A predilection of type for site has been noted in previous work (Riser et al., 1978).

The isolation of klebsiella from patients who are ill does not necessarily implicate these organisms as the causative agents. However, evaluation of the clinical observations and laboratory reports by a paediatrician in the unit using agreed criteria should provide a reasonable assessment of whether an isolate is more likely to be clinically significant, possibly significant, or not relevant.

A single capsular type is usually predominant at any one time when larger numbers are involved. In this case, type 8 was observed for nine months. Then, after a short gap, it appeared again but was surpassed by type 68 . After another interval type 13 replaced the other two. The dominance of any one type may be due to its presence in large enough numbers to prevent other types from becoming established.

The appearance of type 68 may have been due to its introduction at a time when isolations of type 8 were becoming less common. The sudden increase of both may have been related to an outbreak of necrotising enterocolitis which occurred in the SCBU at the same time, especially as type 68 was shown to have a preference for the intestinal tract. The epidemic was attributed to Clostridium butyricum (Howard et al., 1977).

Of the babies from whom klebsiella was initially isolated from the intestinal tract, $30.8 \%$ later yielded the same capsular type from other sites. This figure becomes $\mathbf{4 0} \%$ if untyped klebsiella is also included; ie, in the additional $10 \%$ of cases, it is not known whether the intestinal type is the same as that found in other sites. But experience has shown that isolations from multiple sites in the same patient are almost always of the same capsular type (Riser et al., 1978). These figures approach the $45 \%$ found by Eickhoff (1970) and Selden et al. (1971) and indicate that, in neonates as well as in adults, a 
klebsiella which is first established in the intestinal tract can later be isolated from other sites as either a colonisation or an infection.

When antibiotics were given to babies, $50 \%$ of them developed an infection with klebsiella, while only $8.3 \%$ developed a klebsiella sepsis if they had not received antibiotics. Other factors have not been correlated with this observation; however, several authors have reported that the use of antibiotics may select for Gram-negative bacilli which are often infectious (Selden et al., 1971; Finland, 1972; Terman et al., 1972; Schimpff et al., 1974; Marples, 1975).

In addition to antibiotic usage, certain antiseptics can also partially account for the present emphasis on Gram-negative bacilli in baby units (Stratford, 1963; Forfar et al., 1968). In the SCBU there was standard use of compounds containing hexachlorophane on the babies, including Ster-zac powder, Infacare, and Physohex. Of the three main serotypes $39 \%$ were isolated from the skin and $8.2 \%$, and possibly $24.5 \%$, caused sepsis. Thus klebsiella skin colonisation and infection might be enhanced by the use of this antiseptic.

An investigation of the environment of the SCBU at a time when type 8 was prevalent indicated that all the surfaces, feeds, disinfectants, baby care materials, and fomites, as well as the doctors, nurses, and parents of the babies, were completely free of this klebsiella. The only equipment from which this organism was isolated were incubators and a cot, and these usually yielded the same capsular type that was predominant among the babies at the time.

Records revealed a $47.1 \%$ failure rate of $1 \%$ Hycolin to disinfect the incubators in the SCBU. Of the post-cleaning swabs, $11.8 \%$ grew a klebsiella and $7.8 \%$ of the swabs grew a capsular type 8 klebsiella. Growth from incubator swabs was usually scanty (less than 10 colonies per swab) and often required initial incubation in an enrichment medium.

Kelsey (1975) and Ludescher et al. (1976) have reported the potential hazards of cross-contamination by incubators after use by infected babies and contamination by Gram-negative rods which multiply on moist surfaces.

All of the nurses and mothers who were tested in this study had washed their hands before handling the babies. Of the $43.5 \%$ who had the klebsiella (or an enterobacter) on their hands after changing a baby, $30 \%$ still had the same organism after careful washing with Hibiscrub or Betadine. In all instances the capsular type recovered from the hands was the same as that of the baby being changed. Therefore, in about one out of eight times $(13 \%)$ that a nurse changed an intestinally infected or colonised baby. she still had the organism on her hands as she proceeded to the next stage of care or to the next infant. As many as 64 colonies were recovered from the left and right finger impressions of a nurse after changing a colonised infant.

Fingertip counts should not be regarded as the total number of organisms. The procedure is a convenient means of detecting the presence of bacteria on a site which is easily contaminated, unlikely to be thoroughly cleaned, and from which organisms can be widely disseminated. The actual counts would cover a larger surface area and would be substantially higher. Meticulous handwashing, as conducted during this study, removed most of the klebsiellae, but under the pressure of a busy routine hand washing can be minimal or even forgotten. It was confirmed by the nursing staff that they do not always have time to wash their hands as required.

An oral route for intestinal colonisation has been proposed by Cooke et al. (1969). Shooter et al. (1971) determined that approximately $10^{6}$ Pseudomonas aeruginosa and $10^{5}$ to $10^{6}$ Escherichia coli were necessary for colonisation of healthy adults.

The number of klebsiellae necessary for colonisation of premature infants has not been determined. This patient group may be relatively bacteria-free when exposed to an epidemic strain and may require considerably fewer bacteria for colonisation although breast milk should confer some protection. However, most of the infants were at least partially on artificial feeds.

One nurse was found to have more than 30 colonies on her fingertips after she had fed an infant. This had been preceded by a changing and hand washing.

The epidemic klebsiella was present on nurses' and mothers' hands in larger numbers than were recovered from the contaminated incubators. While it is conceivable that the klebsiella in the incubators could have been a source of infection of the infants, it seems unlikely in this situation. Incubator swabs were generally taken after the occupants had been discharged from the unit, and contaminated incubators were not used until cultures were negative. If an incubator showed the presence of potentially pathogenic organisms while it was being used, the baby was removed immediately. Also, Kelsey (1975) states that the mere recovery of organisms from incubators does not necessarily imply that infection will result.

Colonised babies can provide the main reservoir for a nursery infection (Adler et al., 1970; Bettleheim and Lennox-King, 1976; O'Farrell et al., 1976; Cichon et al., 1977), and many workers have 
attributed the spread of klebsiella between patients to transmission via the hands of the staff (Adler et al., 1970; Barter and Hudson, 1974; Knittle et al., 1975; Casewell et al., 1977; Cichon et al., 1977).

It therefore appears more probable that the organisms isolated from the incubators were a reflection of the type that was infecting the babies, that the infants themselves were the reservoir, and that inadequate handwashing was responsible for spreading the bacteria which resulted in subsequent outbreaks. Further transmission of types 8 and 68 may have been interrupted by the removal of the baby reservoir when the SCBU was closed.

Forty-seven per cent of the nurses and $40 \%$ of the mothers had the same organism on their hands as was present in the intestine of the baby they had just changed. Nurses were undoubtedly more significant in hand transmission than mothers as the former performed more procedures involving a greater number of infants. However, the mothers could have contributed on a smaller scale.

In the Royal Free Hospital, mothers are allowed to handle, change, and feed their premature babies as we believe in the importance of mother-child bonding. However, the mothers did not seem to display sufficient knowledge of the careful hygiene that should be practised when infected, premature infants are being handled.

The following observations were made: After a mother had changed her baby she replaced the materials in the cabinet under the baby's incubator or cot before she washed her hands. A small disposable aluminium container was used for holding water for washing during the changing. This was emptied and rinsed with water in the sink, stored in the cabinet until the next use, and replaced only once every 24 hours. It was observed lying on its side on clean linen and being used as a convenient receptacle for other materials, such as a packet of sterile cotton balls and a jar of zinc and castor oil cream. In two samplings, droplets of water remaining in the containers after a changing revealed the presence of the klebsiella capsular type of that particular baby.

The water taps in the ward were designed to be turned off and on by pushing or pulling with the forearm. One mother was seen to turn on the water by pulling the ends of the bars with her unwashed hands, then, after scrubbing, carefully turn off the water by pushing back the same site with her 'clean' hands. Moreover, mothers have been observed to handle and comfort babies other than their own. According to the staff, hygienic measures are not explained to the mothers. These observations suggest that this could be an unsuspected contribution to cross-infection. Shinebaum et al. (1979) also report $24 \%$ contamination of nurses' and $57 \%$ of mothers' hands with $K$. aerogenes in a study to find sources of neonatal colonisation.

Many hospitals have recognised the importance of establishing an education programme for professional and ancillary groups to train them in hospital epidemiology, surveillance, and control procedures (Hewitt and Sanford, 1974; Dixon, 1975). Rigid rules of hygiene for attendants and equipment are considered more important than the wide use of antibiotics (Davies, 1971; Cichon et al., 1977).

Schimpff et al. (1974) and Gehlbach et al. (1975) suggest the reduction of non-essential staff from a high-risk area and emphasise the importance of decreasing the exposure of infants to visitors and hospital personnel and enforcing aseptic techniques in a nursery.

It is therefore recommended that mothers attending their premature and often infected babies in an intensive care area should be instructed in the necessary hygienic precautions. In addition. such procedures should be re-emphasised continually to the nursing and medical staff to remind them of the microbial aspects of caring for these highly susceptible patients.

We thank Miss Amrat Bhamra for extensive help in isolating the Klebsiella species in the environmental survey.

\section{References}

Adler, J. L., Shulman, J. A., Terry, P. M., Feldman, D. B., and Skaliy, P. (1970). Nosocomial colonization with kanamycin-resistant Klebsiella pneumoniae, types 2 and 11 , in a premature nursery. Journal of Pediatrics, 77, 376-385.

Barter, R. A., and Hudson, J. A. (1974). Bacteriological findings in perinatal pneumonia. Pathology, 6, 223-230.

Bettelheim, K. A., and Lennox-King, S. M. J. (1976). The acquisition of Escherichia coli by new-born babies. Infection, 4, 174-179.

Casewell, M. W., Webster, M., Dalton, M. T., and Phillips, I. (1977). Gentamicin-resistant Klebsiella aerogenes in a urological ward. Lancet, 2, 444-446.

Cichon, M. J., Craig, C. P., Sargent, J., and Brauner, L. (1977). Nosocomial Klebsiella infections in an intensive care nursery. Southern Medical Journal, 70, 33-35.

Cooke, E. M., Ewins, S., and Shooter, R. A. (1969). Changing faecal population of Escherichia coli in hospital medical patients. British Medical Journal, 4, 593-595.

Cowan, S. T. ed. (1974). Cowan and Steel's Manual for the Identification of Medical Bacteria, 2nd edition. Cambridge University Press, London.

Davies, P. A. (1971). Bacterial infection in the fetus and newborn. Archives of Disease in Childhood, 46, 1-27. 
Dixon, R. E. (1975). Techniques for prevention of nosocomial surgical infections (Letter). Journal of the American Medical Association, 231, 1290.

Eickhoff, T. C. (1970). Microbiological sampling. Hospitals, 44, (8) 86-87.

Finland, M. (1972). Changing patterns of susceptibility of common bacterial pathogens to antimicrobial agents. Annals of Internal Medicine, 76, 1009-1036.

Forfar, J. O., Gould, J. C., and Maccabe, A. F. (1968). Effect of hexachlorophane on incidence of staphylococcal and Gram negative infection in the newborn. Lancet, 2, 177-180.

Gehlbach, S. H., Gutman, L. T., Wilfert, C. M., Brumley, G. W., and Katz, S. L. (1975). Recurrence of skin disease in a nursery: ineffectuality of hexachlorophene bathing. Pediatrics, 55, 422-424.

Hable, K. A., Matsen, J. M., Wheeler, D. J., Hunt, C. E., and Quie, P. G. (1972). Klebsiella type 33 septicemia in an infant intensive care unit. Journal of Pediatrics, 80, 920-924.

Hewitt, W. L., and Sanford, J. P. (1974). Workshop on hospital-associated infections. Journal of Infectious Diseases, 130, 680-686.

Howard, F. M., Flynn, D. M., Bradley. J. M., Noone, P., and Szawatkowski, M. (1977). An outbreak of necrotising enterocolitis caused by Clostridium butyricum. Lancet, 2, 1099-1102.

Kelsey, J. C. (1975). Hygiene of babies' incubators. (Letter). Lancet, 1 (7904), 455-456.

Kelsey, J. C., and Maurer, I. M. (1966). An in-use test for hospital disinfectants. Monthly Bulletin of the Ministry of Health and Public Health Laboratory Service, 25, 180-184.

Knittle, M. A., Eitzman, D. V., and Baer, H. (1975). Role of hand contamination of personnel in the epidemiology of Gram-negative nosocomial infections. Journal of Pediatrics, 86, 433-437.

Ludescher, E., Breitfellner, G., and Troy, E. (1976). Klinisch und epidemiologisch ungewöhnliche Infection mit $E$. coli 0111 : B4 auf einer Neugeborenenabteilung. Pädiatrie und Pädologie, 11, 428-434.

Marples, R. R. (1975). Ecology of diseased skin. Journal of Medical Microbiology, 8, p i-ii (Abstract).
O'Farrell, S. M., Lennox-King, S. M. J., Bettelheim, K.A., Shaw, E. J., and Shooter, R. A. (1976). Escherichia coli in a maternity ward. Infection, 4, 146-152.

Pierog, S., Nigam, S., Lala, R. V., Crichlow, D. K., and Evans, H. E. (1977). Neonatal septicemia due to Klebsiella pneumoniae type 60 . Epidemic of unusually low virulence. New York State Journal of Medicine, 77, 737-741.

Riser, E., Noone, P., and Poulton, T. A. (1976). A new serotyping method for Klebsiella species: development of the technique. Journal of Clinical Pathology, 29, 296-304.

Riser, E., Noone, P., and Thompson, R. E. M. (1978). The use of a fluorescence typing method in an epidemiological study of Klebsiella infection in a London hospital. Journal of Hygiene, Cambridge, 80, 43-56.

Schimpff, S. C., Miller, R. M., Polakavetz, S., and Hornick, R. B. (1974). Infection in the severely traumatized patient. Annals of Surgery, 179, 352-357.

Selden, R., Lee, S., Wang, W. L. L., Bennett, J. V., and Eickhoff, T. C. (1971). Nosocomial Klebsiella infections: intestinal colonization as a reservoir. Annals of Internal Medicine, 74, 657-664.

Shinebaum, R., Cooke, E. M., and Brayson, J. C. (1979). Acquisition of Klebsiella aerogenes by neonates. Journal of Medical Microbiology, 12, 201-205.

Shooter, R. A., Faiers, M. C., Cooke, E. M., Breaden, A. L., and O'Farrell, S. M. (1971). Isolation of Escherichia coli, Pseudomonas aeruginosa and Klebsiella from food in hospitals, canteens, and schools. Lancet, 2, 390-392.

Stratford, B. C. (1963). The investigation and treatment of recurrent superficial staphylococcal infection. Medical Journal of Australia, 1, 308-312.

Terman, J. W., Alford, R. H., and Bryant, R. E. (1972). Hospital-acquired Klebsiella bacteremia. American Journal of the Medical Sciences, 264, 191-196.

Requests for reprints to: Dr Paul Noone, The Royal Free Hospital, Pond Street, Hampstead, London NW3 2QG, UK. 